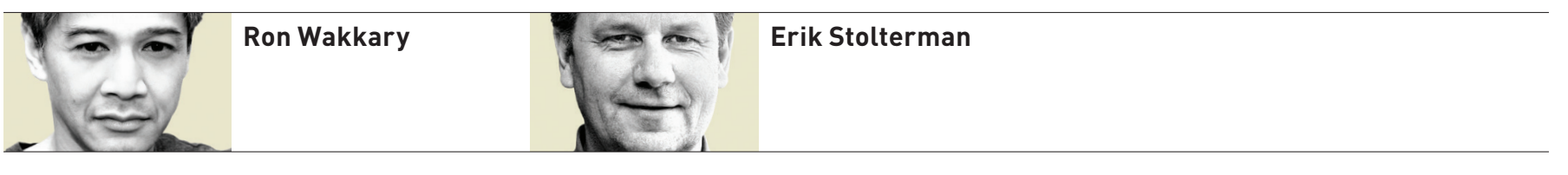

\title{
What's in the Details?
}

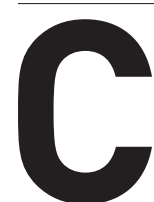

HI 2014 will soon be

here-maybe you are even reading this at the conference in Toronto. The CHI conference is the major event of the year for HCI, for academics as well as for many professionals. CHI is constantly growing. Last year the conference hosted about 3,500 participants in Paris. But CHI is not only the biggest HCI conference, it is also the broadest. It covers almost all aspects of HCI. Our ambition with Interactions is to reflect the full breadth of our field, both as an academic discipline and as a professional practice. This is, of course, not always possible since what constitutes contemporary research and practice in $\mathrm{HCI}$ is constantly changing and evolving. $\mathrm{CHI}$ is for us an important venue since it captures, almost in real time, what people in this broad field are working on. It is the place where trends, developments, and news show up. If you have ideas about how Interactions could better reflect the whole field, please let us know.

HCI covers large questions and problems, designs, and solutions. For instance, in this magazine we regularly publish articles about sustainability, culture, legislation, business, and other big topics. But HCI is also about closely examining the tools of our trade. In this issue's cover story, the focus is on the notion of buttons. Lars-Erik Janlert from Umeå University has carefully studied buttons of all varieties - physical, virtual, simple, and complex. In the article he offers a detailed analysis and conceptual understanding of buttons. This type of deep analysis not only contributes to our understanding of the small details but also challenges our intuitive sense that something small is simple, too.

We also want to remind you to visit the Interactions website (http://interactions.acm.org/). The site is growing and includes an active blog section, where a diverse group of thinkers from our field constantly present their newest ideas and experiences.

As usual, we would like to invite you to write for Interactions. We are always looking for manuscripts that would be suitable for a broader audience.

\section{-Ron Wakkary and Erik Stolterman} eic@interactions.acm.org

\section{Our ambition with Interactions is to reflect the full breadth of our field, both as an academic discipline and as a professional practice.}

